\title{
New Bounds for Zagreb Eccentricity Indices
}

\author{
Nilanjan De \\ Department of Basic Sciences and Humanities (Mathematics), Calcutta Institute of Engineering and Management, Kolkata, India \\ Email: de.nilanjan@rediffmail.com
}

Received August 3, 2012; revised September 3, 2012; accepted October 2, 2012

\begin{abstract}
The Zagreb eccentricity indices are the eccentricity version of the classical Zagreb indices. The first Zagreb eccentricity index $\left(E_{1}(G)\right)$ is defined as sum of squares of the eccentricities of the vertices and the second Zagreb eccentricity index $\left(E_{2}(G)\right)$ is equal to sum of product of the eccentricities of the adjacent vertices. In this paper we give some new upper and lower bounds for first and second Zagreb eccentricity indices.
\end{abstract}

Keywords: Vertex Degree; Eccentricity; Zagreb Eccentricity Indices

\section{Introduction}

Let $G$ be a simple connected graph with vertex set $V(G)$ and edge set $E(G)$ so that $|V(G)|=n$ and $|E(G)|=m$. For any vertex $v \in V(G)$, let $\operatorname{deg}(v)$ denote the degree of the vertex $v$. For vertices $u$ and $v \in V(G)$, the distance between $u$ and $v$ is defined as length of the shortest path connecting $u$ and $v$ and is denoted by $d(u, v)$. The eccentricity of a vertex $v \in V(G)$, denoted by $\varepsilon(v)$, is the distance between $v$ and a vertex farthest from $v$ i.e. $\varepsilon(v)=\max \{d(v, x): x \in V(G)\}$. The radius $r=r(G)$ and diameter $d=d(G)$ of a graph is the minimum and maximum eccentricity among the vertices of $G$ i.e.

$$
r=r(G)=\min \{\varepsilon(v): v \in V(G)\}
$$

and

$$
d=d(G)=\max \{\varepsilon(v): v \in V(G)\}
$$

respectively. Also the total eccentricity of a graph, denoted by $\theta(G)$, is the sum of all the eccentricities of $G$ [1] i.e.

$$
\theta(G)=\sum_{v \in V(G)} \varepsilon(v)
$$

The first and second Zagreb index of a graph were first introduced by Gutman in [2] which are the most known and widely used topological indices, defined as respectively

$$
\begin{aligned}
& M_{1}(G)=\sum_{v \in V(G)} d(v)^{2}, \\
& M_{2}(G)=\sum_{(u, v) \in E(G)} d(u) d(v)
\end{aligned}
$$

Recently several Graph invariants based on vertex eccentricities subject to large number of studies. Analogues to Zagreb indices M. Ghorbani et al. [3] and D. Vukiče- vić et al. [4] defined the Zagreb eccentricity indices by replacing degrees by eccentricity of the vertices. Thus the first and second Zagreb eccentricity indices of a graph $G$ are defined as

$$
\begin{aligned}
& E_{1}(G)=\sum_{v \in V(G)} \varepsilon(v)^{2}, \\
& E_{2}(G)=\sum_{(u, v) \in E(G)} \varepsilon(u) \varepsilon(v)
\end{aligned}
$$

The lower and upper bounds of $n$-vertex trees with fixed diameter and matching number and extremal trees with respect to Zagreb eccentricity indices were studied by R. Xing et al. [5] and recently K. C. Das et al. in [6] presented some properties, upper and lower bounds of Zagreb eccentricity indices and also characterize the extremal graphs.

Another useful eccentricity and degree based topological index called eccentric connectivity index was first introduced by Sharma, Goswami and Madan [7] and is defined as

$$
\begin{aligned}
\xi^{c}(G) & =\sum_{v \in V(G)} \operatorname{deg}(v) \varepsilon(v) \\
& =\sum_{(u, v) \in E(G)}[\varepsilon(u)+\varepsilon(v)]
\end{aligned}
$$

There was a vast research regarding various properties of this topological index [8-10].

The study of determining extremal properties such as upper bounds and lower bounds of some graph invariants were subject to a large number of investigations [11-15]. The aim of this paper is to study similar extremal properties for Zagreb eccentricity indices. In this paper we present some new upper and lower bounds of Zagreb eccentricity indices in terms of number of vertices $(n)$, number of edges $(m)$, radius $(r)$, diameter $(d)$, total eccentricity 
$(\theta(G))$, the first Zagreb indices $\left(M_{1}(G)\right)$, the second Zagreb indices $\left(M_{2}(G)\right)$ and the eccentric eccentricity index $\xi^{c}(G)$.

\section{Bounds for the First Zagreb Eccentricity Index}

We now give some lower and upper bounds of first Zagreb eccentricity index. In [6] Das et al. proved the following upper bound of $E_{1}(G)$.

Theorem 2.1. Let $G$ be a simple connected graph with $n$ vertices and $m$ edges, then

$$
E_{1}(G) \leq M_{1}(G)-4 m n+n^{3}
$$

with equality if and only if $G \cong P_{4}$ or $G \cong K_{n}$ or $G$ is isomorphic to a $(n-1, n-2)$-semiregular graph.

Also, in [5] Xing et al. give the following result.

Theorem 2.2. Let $G$ be a simple connected graph with $n$ vertices and $m$ edges, then

$$
n r^{2} \leq E_{1}(G) \leq n d^{2}
$$

with equality if and only if $G$ all the vertices of $G$ are of same eccentricity.

Also in [6] Das et al. give lower bounds for $E_{1}(G)$ in terms of $n$ and $d$. Now we prove some new upper and lower bounds of $E_{1}(G)$.

Theorem 2.3. Let $G$ be a simple connected graph with $n$ vertices and $m$ edges, then

$$
E_{1}(G) \geq \frac{\theta(G)^{2}}{n}
$$

with equality if and only if $G$ all the vertices of $G$ are of same eccentricity.

Proof Using the Cauchy-Schwartz inequality, we get

$$
\begin{aligned}
& {\left[\varepsilon\left(v_{1}\right)^{2}+\varepsilon\left(v_{2}\right)^{2}+\cdots+\varepsilon\left(e_{n}\right)^{2}\right]\left[1^{2}+1^{2}+\cdots+1^{2}\right]} \\
& \geq\left[\varepsilon\left(v_{1}\right) \cdot 1+\varepsilon\left(v_{2}\right) \cdot 1+\cdots+\varepsilon\left(e_{n}\right) \cdot 1\right]^{2}
\end{aligned}
$$

and hence using the definition of total eccentricity index and first Zagreb eccentricity index the desired result follows. Clearly in the above inequality equality holds when all the vertices of $G$ are of same eccentricity.

Theorem 2.4. Let $G$ be a simple connected, then

$$
E_{1}(G) \geq \frac{M_{1}(G)}{(n-1)^{2}}
$$

with equality if and only if $G \cong K_{n}$.

Proof We have, for all $v \in V(G), \varepsilon(v) \geq \frac{D(v)}{n-1}$, with equality if an only if $G \cong K_{n}$, where $D(v)$ is the degree distance of the vertex $v$ and is defined as

$$
D(v)=\sum_{u \in V(G)} d(v, u) .
$$

Hence from the definition of first Zagreb eccentricity index, we can write

$$
E_{1}(G) \geq \frac{1}{(n-1)^{2}} \sum_{v \in V(G)} D(v)^{2}
$$

Now since $D(v) \geq \operatorname{deg}(v)$ for all $v \in V(G)$, so from (2.1) we get the desired result with equality if and only if $\operatorname{deg}(v)=(n-1)$ and $\varepsilon(v)=1$, that is $G \cong K_{n}$.

Corollary 2.1. Let $G$ be a simple connected graph with $n$ vertices and $m$ edges, then

$$
E_{1}(G) \geq \frac{M_{1}(G)}{(n-1)^{2}}-\frac{8 m}{n-1}+4 n
$$

with equality if and only if $G$ is a path of length one.

Proof Again since, $D(v) \geq 2 n-2-\operatorname{deg}(v)$, for all $v \in V(G)$, with equality if and only if $G \cong K_{1, n-1}$, so from (2.1) we get the desired result. Since the equality (2.1) holds if and only if $G \cong K_{n}$ so the equality holds in this result if and only if $G$ is a path of length one which is the only complete graph as well as complete bipartite graph.

Theorem 2.5. Let $G$ be a simple connected graph on $n$ vertices and $n_{0}$ be the number of vertices with eccentricity one in $G$, then

$$
E_{1}(G) \geq\left(4 n-3 n_{0}\right)
$$

with equality if and only if $G \cong K_{n}-\frac{n-n_{0}}{2}$, where $\left(n-n_{0}\right)$ is even.

Proof Since $n_{0}$ be the number of vertices with eccentricity one in $G$, so the remaining $\left(n-n_{0}\right)$ vertices are of eccentricity at least two. Let $S=\left\{v_{i}: 1 \leq i \leq n_{0}\right\}$ be the set of vertices such that $\varepsilon\left(v_{i}\right)=1$ for $i=1,2, \cdots, n_{0}$. Then from the definition of first Zagreb eccentricity index, we have

$$
E_{1}(G)=n_{0}+\left(n-n_{0}\right) \sum_{u \in V(G) \backslash S} \varepsilon(u)^{2} \geq n_{0}+4\left(n-n_{0}\right)
$$

from where the desired result follows. Clearly, in this theorem equality holds if and only if $G \cong K_{n}-\frac{n-n_{0}}{2}$, where $\left(n-n_{0}\right)$ is even.

Theorem 2.6. Let $G$ be a simple connected graph with $n$ vertices and $m$ edges, then

$$
E_{1}(G) \geq(r+d) \theta(G)-n r d
$$

with equality if and only if all the vertices of $G$ are of same eccentricity.

Proof To prove this theorem, using the following Diaz-Metcalf inequality, we have, if $a_{i}$ and $b_{i}$, $i=1,2, \cdots, n$ are real numbers such that $h a_{i} \leq b_{i} \leq H a_{i}$ for $i=1,2, \cdots, n$, then 


$$
\sum_{i=1}^{n} b_{i}^{2}+h H \sum_{i=1}^{n} a_{i}^{2} \leq(h+H) \sum_{i=1}^{n} a_{i} b_{i}
$$

In the above inequality equality holds if and only if $b_{i}=h a_{i}$ or $b_{i}=H a_{i}$ for every $i=1,2, \cdots, n$. By setting $b_{i}=\varepsilon\left(v_{i}\right)$ and $a_{i}=1$, for $i=1,2, \cdots, n$, in (2.2) from above inequality we get

$$
\sum_{i=1}^{n} \varepsilon\left(v_{i}\right)^{2}+h H \sum_{i=1}^{n} 1^{2} \leq(h+H) \sum_{i=1}^{n} \varepsilon\left(v_{i}\right)
$$

Now using the definition of first Zagreb eccentricity index and total eccentricity index we get

$$
E_{1}(G) \leq(h+H) \theta(G)-h H n
$$

Since, $r \leq \varepsilon\left(v_{i}\right) \leq d$ for $i=1,2, \cdots, n$, so we have $h=r$ and $H=d$. Hence the desired result follows from above. Clearly in the above inequality equality holds if and only if all the vertices of $G$ are of same eccentricity.

Theorem 2.7. Let $G$ be a simple connected graph, then

$$
E_{1}(G) \leq(n+r-1) \theta(G)-n(n-1) r
$$

In the above inequality equality holds if and only if all the vertices of $G$ are of same eccentricity.

Proof Let, sum of eccentricities of the vertices adjacent to

$$
\begin{aligned}
v_{i} & =\varepsilon\left(v_{i}\right) m_{i}=\sum_{\left(v_{i}, v_{j}\right) \in E(G)} \varepsilon\left(v_{j}\right) \\
& \leq \theta(G)-\varepsilon\left(v_{i}\right)-\left(n-1-\varepsilon\left(v_{i}\right)\right) r
\end{aligned}
$$

so that

$$
\begin{aligned}
E_{1}(G) & =\sum_{v_{i} \in V(G)} \varepsilon\left(v_{i}\right)^{2}=\sum_{i=1}^{n} \varepsilon\left(v_{i}\right) m_{i} \\
& \leq n \theta(G)-\theta(G)-n(n-1) r+r \theta(G)
\end{aligned}
$$

from where we get the desired result. Obviously in the above inequality equality holds if and only if all the vertices of $G$ are of same eccentricity.

Theorem 2.8. Let $G$ be a simple connected graph on $n$ vertices and $m$ edges, then

$$
E_{1}(G) \geq n \theta(G)\left[\frac{\theta(G)-r(n-1)}{\theta(G)-n(r-1)}\right]
$$

In the above inequality equality holds if and only if all the vertices of $G$ are of same eccentricity.

Proof We will prove this theorem using the following Chebyschev's inequality: Let $a_{i}$ and $b_{i}$ are real numbers, then

$$
n \sum_{i=1}^{n} a_{i} b_{i} \geq \sum_{i=1}^{n} a_{i} \sum_{i=1}^{n} b_{i}
$$

with equality holds if and only if

$$
a_{1}=a_{2}=\cdots=a_{n} \text { or } b_{1}=b_{2}=\cdots=b_{n} .
$$

Now setting $a_{i}=\varepsilon\left(v_{i}\right)$ and $b_{i}=\varepsilon\left(v_{i}\right) m_{i}$, for $i=1,2, \cdots, n$, we get from (2.3)

$$
\begin{aligned}
n \sum_{i=1}^{n} \varepsilon\left(v_{i}\right) \varepsilon\left(v_{i}\right) m_{i} & \geq \sum_{i=1}^{n} \varepsilon\left(v_{i}\right) \sum_{i=1}^{n} \varepsilon\left(v_{i}\right) m_{i} \\
& =\theta(G) E_{1}(G)
\end{aligned}
$$

Again since

$$
\sum_{i=1}^{n} \varepsilon\left(v_{i}\right) m_{i} \leq \theta(G)-n \varepsilon\left(v_{i}\right)-\left(n-1-\varepsilon\left(v_{i}\right)\right) r
$$

with equality holding if and only if

$$
\varepsilon\left(v_{1}\right)=\varepsilon\left(v_{2}\right)=\cdots=\varepsilon\left(v_{n}\right),
$$

we get from (2.4)

$$
\begin{aligned}
\theta(G) E_{1}(G) & \leq n \theta(G)^{2}-n r(n-1) \theta(G) \\
& +n(r-1) E_{1}(G)
\end{aligned}
$$

from where we get the desired result. Clearly in this inequality equality holds if and only if all the vertices are of same eccentricity.

Theorem 2.9. Let $G$ be a simple connected graph where all the vertices must not be of equal eccentricity, then

$$
E_{1}(G) \leq(d+r) \theta(G)-n d r-(n-a-b)(d-r-1)
$$

where $G$ consists of a number of vertices with eccentricity $d$ and $b$ number of vertices with eccentricity $r$. In the above inequality equality holds if and only if eccentricities of the vertices are equal to $r+1, r, d-1, d$.

Proof For any vertex $v_{i} \in V(G)$, we have

$$
\begin{aligned}
(d-r-1) & \leq\left(d-\varepsilon\left(v_{i}\right)\right)\left(\varepsilon\left(v_{i}\right)-r\right) \\
& =-\varepsilon\left(v_{i}\right)^{2}+(d+r) \varepsilon\left(v_{i}\right)-r d
\end{aligned}
$$

with equality holds if and only if $\varepsilon\left(v_{i}\right)=r+1$ or $\varepsilon\left(v_{i}\right)=d-1$ for $i=1,2, \cdots, n$. Now summing the above inequality for $r<\varepsilon\left(v_{i}\right)<d$, we get

$$
\begin{aligned}
\sum_{r<\varepsilon\left(v_{i}\right)<d} \varepsilon\left(v_{i}\right)^{2} \leq & -(n-a-b)(d-r-1)-(n-a-b) r d \\
& +(d+r)(\theta(G)-a d-b r)
\end{aligned}
$$

So,

$$
\begin{aligned}
E_{1}(G)= & \sum_{r<\varepsilon\left(v_{i}\right)<d} \varepsilon\left(v_{i}\right)^{2}+a d^{2}+b r^{2} \leq a d^{2}+b r^{2} \\
& -(n-a-b)(d-r-1)-n r d+(a+b) r d \\
& +\theta(G)(d+r)-(a d+b r)(d+r)
\end{aligned}
$$

from where we get the desired result. In the above inequality equality holds if and only if

$$
\varepsilon\left(v_{i}\right) \in\{r, r+1, d-1, d\} .
$$

From the above theorem the following result directly follows. 
Corollary 2.2. Let $G$ be a simple connected graph with $n$ vertices and $m$ edges, then

$$
\begin{aligned}
E_{1}(G) & \leq(d+r) \theta(G)-n d r \\
& -(n-k)(d-r-1)
\end{aligned}
$$

where $k$ is the number of vertices having eccentricity equal to $d$ or $r$.

\section{Bounds for the Second Zagreb Eccentricity Index}

In [6] Das et al. give lower bounds for $E_{2}(G)$ in terms of $m, d$ and proved the following upper bound of $E_{1}(G)$.

Theorem 3.1. Let $G$ be a simple connected graph with $n$ vertices and $m$ edges, then

$$
E_{2}(G) \leq M_{2}(G)-n M_{1}(G)+m n^{2}
$$

with equality if and only if $G \cong P_{4}$ or $G \cong K_{n}$ or $G$ is isomorphic to a $(n-1, n-2)$-semiregular graph.

Also, in [5] Xing et al. proved the following result.

Theorem 3.2. Let $G$ be a simple connected graph with $n$ vertices and $m$ edges, then

$$
m r^{2} \leq E_{2}(G) \leq m d^{2}
$$

with equality if and only if $G$ all the vertices of $G$ are of same eccentricity.

Now we prove some new upper and lower bounds of $E_{2}(G)$.

Theorem 3.3. Let $G$ be a simple connected graph with $n$ vertices and $m$ edges, then

$$
E_{2}(G) \geq \frac{m}{n^{2}} \theta(G)^{2}
$$

with equality if and only if $G$ all the vertices of $G$ are of same eccentricity.

Proof Using the inequality between arithmetic and geometric mean, we get

$$
\begin{aligned}
& \frac{1}{m} \sum_{\left(v_{i}, v_{j}\right) \in E(G)} \varepsilon\left(v_{i}\right) \varepsilon\left(v_{j}\right) \\
& \geq\left[\prod_{\left(v_{i}, v_{j}\right) \in E(G)} \varepsilon\left(v_{i}\right) \varepsilon\left(v_{j}\right)\right]^{\frac{1}{m}}=\left[\prod_{i=1}^{m} \varepsilon\left(v_{i}\right)^{d\left(v_{i}\right)}\right]^{\frac{1}{m}}
\end{aligned}
$$

Now let, $P=\prod_{i=1}^{m} \varepsilon\left(v_{i}\right)^{d\left(v_{i}\right)}$, so that taking natural logarithm on both sides and using Jensen's inequality, we get

$$
\begin{aligned}
\ln P & =\sum_{i=1}^{n} d\left(v_{i}\right) \ln \varepsilon\left(v_{i}\right) \geq \sum_{i=1}^{n} d\left(v_{i}\right) \ln \frac{1}{n} \sum_{i=1}^{n} \varepsilon\left(v_{i}\right) \\
& =2 m \ln \frac{\theta(G)}{n}
\end{aligned}
$$

Thus $P \geq \ln \left(\frac{\theta(G)}{n}\right)^{2 m}$, so that $E_{2}(G) \geq \frac{m}{n^{2}} \theta(G)^{2}$,

which is the desired result. In this inequality equality holds if and only if all the vertices of $G$ are of same eccentricity.

Theorem 3.4. Let $G$ be a simple connected graph, then

$$
E_{2}(G) \geq \xi^{c}(G)-m
$$

where, $\xi^{c}(G)$ is the eccentric connectivity index of $G$. In the above inequality equality holds if and only if $\varepsilon(u)=1$ or $\varepsilon(v)=1$ for all $(u, v) \in E(G)$.

Proof Since $\varepsilon(v) \geq 1$ for all $v \in V(G)$, we have $\varepsilon(u) \varepsilon(v) \geq \varepsilon(u)+\varepsilon(v)-1$. So from the definition of second Zagreb eccentricity index, we can write

$$
E_{2}(G)=\sum_{(u, v) \in E(G)} \varepsilon(u) \varepsilon(v) \geq \sum_{(u, v) \in E(G)}[\varepsilon(u)+\varepsilon(v)]-m
$$

Now since

$$
\xi^{c}(G)=\sum_{v \in V(G)} \operatorname{deg}(v) \varepsilon(v)=\sum_{(u, v) \in E(G)}[\varepsilon(u)+\varepsilon(v)],
$$

the desired result follows from above. In the above inequality equality holds if and only if $\varepsilon(u)=1$ or $\varepsilon(v)=1$ for all $(u, v) \in E(G)$, for example if and only if $G \cong K_{n}$ or $G \cong K_{1, n-1}$.

Theorem 3.5. Let $G$ be a simple connected graph with $n$ vertices and $m$ edges, then

$$
E_{2}(G) \geq \frac{M_{2}(G)}{(n-1)^{2}}
$$

with equality if and only if $G \cong K_{n}$.

Proof Since, for all $v \in V(G), \varepsilon(v) \geq \frac{D(v)}{n-1}$, with equality if an only if $G \cong K_{n}$, like Theorem 2.4, using definition of second Zagreb eccentricity index the desired result follows.

Corollary 3.1. Let $G$ be a simple connected graph with $n$ vertices and $m$ edges, then

$$
E_{2}(G) \geq \frac{M_{2}(G)}{(n-1)^{2}}-\frac{2 M_{1}(G)}{n-1}+4 m
$$

with equality if and only if $G$ is a path of length one.

Proof Since, $D(v) \geq 2 n-2-\operatorname{deg}(v)$ for all $v \in V(G)$, with equality if and only if $G \cong K_{1, n-1}$, like Corollary 2.1, from the Theorem 3.5 we have

$$
\begin{aligned}
E_{2}(G) \geq & 4 m-\frac{2}{(n-1)} \sum_{(u, v) \in E(G)}[\operatorname{deg}(u)+\operatorname{deg}(v)] \\
& -\frac{1}{(n-1)^{2}} \sum_{(u, v) \in E(G)}[\operatorname{deg}(u) \operatorname{deg}(v)]
\end{aligned}
$$

from where we get the desired result. Like Corollary 2.1, 
in this inequality equality holds if and only if $G$ is a path of length one.

Theorem 3.6. Let $G$ be a simple connected graph, then

$$
E_{2}(G) \leq m(\theta(G)-n+1)+\frac{1}{2}(r-1) \xi^{c}(G)
$$

In the above inequality equality holds if and only if all the vertices of $G$ are of same eccentricity.

Proof Since,

$$
\varepsilon\left(v_{i}\right) m_{i} \leq \theta(G)-\varepsilon\left(v_{i}\right)-\left(n-1-\varepsilon\left(v_{i}\right)\right) r
$$

for $i=1,2, \cdots, n$, we have

$$
\begin{aligned}
E_{2}(G) & =\frac{1}{2} \sum_{i=1}^{n} \operatorname{deg}\left(v_{i}\right) \sum_{\left(v_{i}, v_{j}\right) \in E(G)} \varepsilon\left(v_{i}\right) \\
& =\frac{1}{2} \sum_{i=1}^{n} \operatorname{deg}\left(v_{i}\right) \varepsilon\left(v_{i}\right) m_{i} \\
& \leq \frac{1}{2}\left[2 m \theta(G)-\xi^{c}(G)-2 m(n-1) r+r \xi^{c}(G)\right]
\end{aligned}
$$

from where we get the desired result. Obviously in the above inequality equality holds if and only if all the vertices of $\mathrm{G}$ are of same eccentricity.

Corollary 3.2. Let $G$ be a simple connected graph with $n$ vertices and $m$ edges, then

$$
E_{1}(G) \geq m \theta(G)-\frac{1}{2}(r-1) M_{1}(G)+m(n r-2 n+1)
$$

with equality if and only if $G \cong K_{n}$ or $G$ is obtained from $K_{n}$ by removing a perfect matching.

Proof Since we have [8], $\xi^{c}(G) \leq 2 m n-M_{1}(G)$, with equality if and only if $G \cong K_{n}-k e$ for $k=0,1,2, \cdots,\lfloor n / 2\rfloor$ or $G \cong P_{4}$, from the above theorem the desired result follows.

\section{Conclusion}

In this paper we have established some sharp upper and lower bounds of the Zagreb eccentricity indices in terms of some graph parameters such as order, size, radius, diameter, eccentric connectivity index, total eccentricity, first and second Zagreb indices. It may be useful to give the bounds for $E_{1}(G)$ and $E_{2}(G)$ indices in terms of other graph invariants.

\section{REFERENCES}

[1] P. Dankelmann, W. Goddard and C. S. Swart, "The Average Eccentricity of a Graph and Its Subgraphs," UtilitasMathematica, Vol. 65, 2004, pp. 41-51.
[2] I. Gutman and N. Trinajstić, "Graph Theory and Molecular Orbitals, Total $\varphi$-Electron Energy of Alternant Hydrocarbons," Chemical Physics Letters, Vol. 17, 1972, pp. 535-538. doi:10.1016/0009-2614(72)85099-1

[3] D. Vukičević and A. Graovac, "Note on the Comparison of the First and Second Normalized Zagreb Eccentricity Indices," Acta Chimica Slovenica, Vol. 57, 2010, pp. 524528.

[4] M. Ghorbani and M. A. Hosseinzadeh, "A New Version of Zagreb Indices," Filomat, Vol. 26, No. 1, 2012, pp. 93100. doi:10.2298/FIL1201093G

[5] R. Xing, B. Zhou and N. Trinajstić, "On Zagreb Eccentricity Indices," Croatica Chemica Acta, Vol. 84, No. 4, 2011, pp.493-497. doi:10.5562/cca1801

[6] K. C. Das, D. W. Lee and A. Gravovac, "Some Properties of Zagreb Eccentricity Indices," Ars Mathematica Contemporanea, Vol. 6, No. 1, 2013, pp. 117-125.

[7] V. Sharma, R. Goswami and A. K. Madan, "Eccentric Connectivity Index: A Novel Highly Discriminating Topological Descriptor for Structure-Property and Structure-Activity Studies," Journal of Chemical Information Computer Sciences, 1997, Vol. 37, No. 2, pp. 273-282. doi:10.1021/ci960049h

[8] B. Zhou and Z. Du, "On Eccentric Connectivity Index," MATCH: Communications in Mathematical and in Computer Chemistry, Vol. 63, No. 1, 2010, pp. 181-198.

[9] N. De, "Eccentric Connectivity Index of Thorn Graph," Applied Mathematics, Vol. 3, No. 8, 2012, pp. 931-934. doi:10.4236/am.2012.38139

[10] A. Ilić and I. Gutman, "Eccentric Connectivity Index of Chemical Trees," MATCH: Communications in Mathematical and in Computer Chemistry, Vol. 65, 2011, pp. 731-744.

[11] K.C. Das, "Maximizing the Sum of the Squares of Degrees of a Graph," Discrete Mathematics, Vol. 285, No. 1-3, 2004, pp. 57-66. doi:10.1016/j.disc.2004.04.007

[12] A. Ilić, M. Ilić and B. Liu, "On the Upper Bounds for the First Zagreb Index," Kragujevac Journal of Mathematics, Vol. 35, No. 1, 2011, pp. 173-182.

[13] N. De, "Some Bounds of Reformulated Zagreb Indices," Applied Mathematical Sciences, Vol. 6, No. 101-104, 2012, pp. 5005-5012.

[14] K. Ch. Das, I. Gutman and B. Zhou, "New Upper Bounds on Zagreb Indices," Journal of Mathematical Chemistry, Vol. 46, No. 2, 2009, pp. 514-521. doi:10.1007/s10910-008-9475-3

[15] N. De, "Bounds for the connective eccentric index," International Journal of Contemporary Mathematical Sciences, Vol. 7, No. 44, 2012, pp. 2161-2166. 